BUTP-95/18

\title{
Fixed point action and topology in the $\mathrm{CP}^{3}$ model
}

\author{
Rudolf Burkhalter \\ Institut für theoretische Physik, Universität Bern, \\ Sidlerstrasse 5, CH-3012 Bern, Switzerland.
}

(December 1995)

\begin{abstract}
We define a fixed point action in two-dimensional lattice $\mathrm{CP}^{N-1}$ models The fixed point action is a classical perfect lattice action, which is expected to show strongly reduced cutoff effects in numerical simulations. Furthermore, the action has scale-invariant instanton solutions, which enables us to define a correct topological charge without topological defects. Using a parametrization of the fixed point action for the $\mathrm{CP}^{3}$ model in a Monte Carlo simulation, we study the topological susceptibility.
\end{abstract}

11.15.Ha 75.10.Hk 11.10.Hi

Typeset using REVTEX 


\section{INTRODUCTION}

One possible way of regularizing a continuum quantum field theory is to introduce a lattice as a UV regulator. An additional benefit of this method is that it opens the door to computer simulations. However, by naively discretizing physical observables, the correct values are only obtained in the continuum limit, when the lattice spacing is going to zero. At finite lattice spacing the lattice induces systematic errors (cutoff effects). In order to remove these cutoff effects one has to introduce finer and finer lattices, and finally extrapolate the calculated quantities to the continuum limit. This delicate limit is a major difficulty in extracting continuum physics from the lattice.

It is possible to circumvent this problem by using Wilson's renormalization group theory. If one constructs the lattice action and all operators in a form that corresponds to the renormalized trajectory, one obtains results that do not depend on the lattice spacing. It has been shown in a series of papers recently published [1,2] that it is possible to construct a fixed point action for asymptotically free theories. This action is the fixed point (FP) of an exact renormalization group $(\mathrm{RG})$ transformation and as such may be taken as a first approximation to the renormalized trajectory.

As a pilot project a local parametrization of the FP action was constructed in the $\mathrm{O}(3)$ nonlinear $\sigma$ model, and used in numerical simulations [四]. The result was very promising: Although the FP action is perfect strictly only classically, no cutoff effects were seen even at small correlation lengths $(\xi \sim 3)$.

In a subsequent paper [3] a FP topological charge was proposed. It was shown, that the combination FP action and FP charge has no topological defects. The FP action has the correct value for instanton solutions, and does not depend on the scale of the instanton. Hence it admits stable instanton solutions on the lattice. This is in contrast to the standard lattice action, whose value depends on the instanton scale, and which suffers from dislocations [4]. These dislocations were suspected to be responsible for the non scaling behavior of the topological susceptibility in the $\mathrm{O}(3)$ nonlinear $\sigma$ model. However, using parametriza- 
tions of the FP action and the FP topological charge in a Monte Carlo simulation, even with the absence of dislocations a strong violation of scaling of the topological susceptibility was established [3]. This indicates that the topological susceptibility is not a physical quantity in the $\mathrm{O}(3)$ nonlinear $\sigma$ model. This observation is supported by results of semiclassical approximations [5.6], where the instanton size distribution is divergent for small instanton sizes.

In $\mathrm{CP}^{N-1}$ models with $N>2$, however, semiclassical approximation indicates that there is no dominance of small instantons. Furthermore, for $N>3$ dislocations are suppressed even for the standard lattice action [4]. It should, therefore, be possible, to determine unambiguously the topological susceptibility in these models. On the other hand, there were several recent determinations of the topological susceptibility in the $\mathrm{CP}^{3}$ model, using different discretizations of the action and the topological charge [7]11; but the results of these determinations are partly in plain contradiction with each other. The situation is therefore by no means clear. We suggest to use the same concepts and methods in order to determine the topological susceptibility as was proposed in Ref. [3].

This paper is organized as follows. In Sec. III we construct a classical perfect lattice action for the $\mathrm{CP}^{3}$ model along the same lines as in Ref. [1]. We define a RG transformation and determine the FP with analytical and numerical methods. We give a parametrization of the FP action, that works reasonably well even for coarse grained fields. In Sec. [V] we define a FP topological charge along the lines of in Ref. [3]. In order to use the FP charge in numerical simulations, we construct a parametrization of the dependence of the fine field on a coarse input field. In Sec. $\nabla$ we discuss the topological susceptibility and the influence of a lattice regularization on its measurement. Finally, we present the results of numerical simulations using the FP action and the FP charge. 


\section{II. $\mathrm{CP}^{\mathrm{N}-1}$ MODELS}

Two-Dimensional $\mathrm{CP}^{N-1}$ models are an important testing ground for methods in quantum field theories because of their similarities with four-dimensional non-Abelian gauge theories. Important common properties are asymptotic freedom, dynamical mass generation, confinement of non-gauge-invariant states and a nontrivial topology. Despite these common features, two-dimensional spin models are much easier to handle both analytically and numerically.

The $\mathrm{CP}^{N-1}$ model (in the continuum) consists of a $N$-component complex spin field $z^{i}(x)$ which satisfies the constraint $\bar{z}(x) \cdot z(x)=1$. The action has a global $\mathrm{SU}(N)$ symmetry and a local $U(1)$ (gauge) symmetry. The gauge field, however, is not independent and can be expressed in the basic field $z(x)$. Only after quantization, the gauge field becomes dynamical, and gives rise to a confining potential, as is explicitly seen in the large- $N$ limit [12]. In the following it will sometimes prove useful, not to use the basic field $z(x)$, but the gauge invariant composite operator $P(x)=z(x) \otimes \bar{z}(x)$. For $N=2$ the $\mathrm{CP}^{N-1}$ model is equivalent to the $\mathrm{O}(3)$ nonlinear $\sigma$ model and $P(x)$ can be written in terms of the usual $\mathrm{O}(3)$ covariant spins $\mathbf{S}(x)$ as $P(x)=\frac{1}{2}(\mathbb{1}+\sigma \cdot \mathbf{S}(x))$. Here $\sigma$ are the usual Pauli matrices. In the notation with the composite operators the continuum action for $\mathrm{CP}^{N-1}$ assumes the form

$$
N \beta \mathcal{A}_{\text {cont }}=\frac{N \beta}{2} \int d^{2} x \operatorname{tr}\left\{\partial_{\mu} P(x) \partial_{\mu} P(x)\right\}
$$

One has several possibilities to discretize the continuum theory, and to put it on a lattice [13]. If one approaches the continuum limit, each of it will give the same results. We choose for this study a discretization, which does not use an explicit gauge field. The standard lattice action without gauge fields assumes the form

$$
N \beta \mathcal{A}_{\mathrm{ST}}=N \beta \sum_{n, \mu}\left\{1-\left|\bar{z}_{n+\hat{\mu}} z_{n}\right|^{2}\right\}
$$

For $N=2$ this goes over to the standard lattice action of the $\mathrm{O}(3)$ nonlinear $\sigma$ model, which is not the case if one would use a formulation with an explicit gauge field. The 
parametrization of the FP action will be a generalization of the standard action, taking into account couplings between two spins, that are more distant than nearest neighbor and also multispin couplings between up to four spins.

\section{FIXED POINT ACTION}

\section{A. Equation for the fixed point action}

We consider the $\mathrm{CP}^{N-1}$ model on a two-dimensional square lattice with variables $z_{n}$ at each lattice site $n$. Then we perform an exact RG transformation. For this we split the lattice into $2 \times 2$ blocks $n_{B}$ and with each block we associate a block spin $\zeta_{n_{B}}$. We define

the (gauge invariant) RG transformation as an averaging over the fine spins in one block, that has the form

$$
e^{-N \beta^{\prime} \mathcal{A}^{\prime}(\zeta)}=\int_{z} \mathcal{N}(z) \exp \left\{-N \beta \mathcal{A}(z)+\kappa N \beta \sum_{n_{B}} \sum_{n \in n_{B}}\left|\zeta_{n_{B}} \bar{z}_{n}\right|^{2}\right\} .
$$

Here, $\kappa$ is a free parameter of the RG transformation, and the normalizing factor $\mathcal{N}(z)$ assures, that the partition function does not change under the RG transformation

$$
\mathcal{N}(z)^{-1}=\int_{\zeta} \exp \left\{\kappa N \beta \sum_{n_{B}} \sum_{n \in n_{B}}\left|\zeta_{n_{B}} \bar{z}_{n}\right|^{2}\right\} .
$$

The form of the RG transformation in Eq. (3) was chosen, such that for the $\mathrm{CP}^{1}$ model it corresponds to the one used for the $\mathrm{O}(3)$ nonlinear $\sigma$ model in Ref. [1]. In order to compute the normalizing factor $\mathcal{N}(z)$, consider the unitary matrix

$$
M_{n_{B}}=\sum_{n \in n_{B}} z_{n} \otimes \bar{z}_{n}
$$

It is just the sum of the composite operators $P_{n}$ at every site of a block. Let the Hermitian matrix $M_{n_{B}}$ have eigenvalues $\lambda_{n_{B}}^{i}$ and eigenvectors $w_{n_{B}}^{i}$. Because $M_{n_{B}}$ is gauge invariant, also its eigenvalues and eigenvectors do not depend on the gauge. Using these eigenvalues and eigenvectors, we can rewrite one term in Eq. (3)

$$
\sum_{n \in n_{B}}\left|\zeta_{n_{B}} \bar{z}_{n}\right|^{2}=\bar{\zeta}_{n_{B}} M_{n_{B}} \zeta_{n_{B}}=\sum_{i} \lambda_{n_{B}}^{i}\left|\zeta_{n_{B}} \bar{w}_{n_{B}}^{i}\right|^{2}
$$


In the limit $\beta \rightarrow \infty$ we perform a saddle point approximation. We get to lowest order $\mathcal{N}(z)^{-1}=\exp \left(\kappa N \beta \sum_{n_{B}} \hat{\lambda}_{n_{B}}\right)$, where $\hat{\lambda}_{n_{B}}$ is the largest eigenvalue of $M_{n_{B}}$. The FP of the $\mathrm{RG}$ transformation (31) is determined in this limit by the implicit equation (FP equation)

$$
\mathcal{A}_{\mathrm{FP}}(\zeta)=\min _{\{z\}}\left\{\mathcal{A}_{\mathrm{FP}}(z)+\mathcal{T}(\zeta, z)\right\}
$$

with the transformation kernel

$$
\mathcal{T}(\zeta, z)=\kappa \sum_{n_{B}}\left(\hat{\lambda}_{n_{B}}-\sum_{n \in n_{B}}\left|\zeta_{n_{B}} \bar{z}_{n}\right|^{2}\right) .
$$

The kernel $\mathcal{T}(\zeta, z)$ is gauge invariant in a strong sense: It does not change under independent gauge transformations on either the coarse or on the fine spins. This is more than would be needed: in a general case it would be sufficient, that the kernel is gauge invariant under a combined gauge transformation on the coarse and the fine spins. The gauge invariance of the kernel assures, that if the action $\mathcal{A}_{\mathrm{FP}}(z)$ of the fine field is gauge invariant, then also the action $\mathcal{A}_{\mathrm{FP}}(\zeta)$ of the coarse field after the RG transformation is gauge invariant.

The FP equation (7) determines the value of the FP action for a given input configuration $\{\zeta\}$. One may solve this equation iteratively, leading to a minimization on a multigrid of lattice configurations with the configuration $\{\zeta\}$ on the coarsest level, and with $k$ finer configurations $\left\{z^{(k)}\right\}$ on successive levels:

$$
\mathcal{A}^{(k)}(\zeta)=\min _{\left\{z^{(1)}, z^{(2)}, \ldots, z^{(k)}\right\}}\left\{\mathcal{A}^{(0)}\left(z^{(k)}\right)+\mathcal{T}\left(z^{(k-1)}, z^{(k)}\right)+\ldots+\mathcal{T}\left(\zeta, z^{(1)}\right)\right\}
$$

On each successive level the spin configurations become smoother and smoother, hence one may choose for the action $\mathcal{A}^{(0)}\left(z^{(k)}\right)$ on the finest configuration $\left\{z^{(k)}\right\}$ any lattice discretization of the continuum action. The FP action $\mathcal{A}_{\mathrm{FP}}(\zeta)$ is then obtained in the limit $k \rightarrow \infty$ of $\mathcal{A}^{(k)}(\zeta)$. For practical purposes, however, only a few levels are needed, and, starting from the standard action on the lowest level, the FP value is reached soon. This iterative method can be used to solve the FP equation numerically. One can, however, make some important statements even without explicitly solving Eq. (7). This will be done in the next section. 


\section{B. Classical Solutions}

As in the $\mathrm{O}(3)$ nonlinear $\sigma$ model we can show the following important statement concerning classical solutions (e.g. instanton solutions).

Statement: If the coarse configuration $\{\zeta\}$ satisfies the FP classical equations of motion (i.e. the classical equations corresponding to $\mathcal{A}_{\mathrm{FP}}$ ), and therefore is a local minimum of $\mathcal{A}_{\mathrm{FP}}(\zeta)$, then the configuration $\{z(\zeta)\}$ on the fine lattice, which minimizes the right handside of Eq. (7), satisfies the equations of motion as well. In addition, the value of the action remains unchanged: $\mathcal{A}_{\mathrm{FP}}(z(\zeta))=\mathcal{A}_{\mathrm{FP}}(\zeta)$.

Proof: Since $\{\zeta\}$ is a solution of the FP classical equations of motion, it is a stationary point of $\mathcal{A}_{\mathrm{FP}}(\zeta)$, and it satisfies $\delta \mathcal{A}_{\mathrm{FP}} / \delta \zeta=0$. The transformation kernel is positive:

$$
\mathcal{T}_{n_{B}}=\hat{\lambda}_{n_{B}}-\bar{\zeta}_{n_{B}} M_{n_{B}} \zeta_{n_{B}} \geq 0
$$

The minimizing configuration $\{z\}$ must fulfill $\mathcal{T}_{n_{B}}=0$, otherwise we could find another coarse configuration $\{\zeta\}$, that fulfills this equation and lowers therefore the value of $\mathcal{A}_{\mathrm{FP}}(\zeta)$; which would be in contradiction to the assumption, that $\{\zeta\}$ is a stationary point. Hence we have a (albeit not unique) relation between the given coarse field $\{\zeta\}$ and the minimizing fine field $\{z(\zeta)\}$

$$
M_{n_{B}} \zeta_{n_{B}}=\hat{\lambda}_{n_{B}} \zeta_{n_{B}}
$$

in other words, $\zeta_{n_{B}}$ is the eigenvector corresponding to the largest eigenvalue of the matrix $M_{n_{B}}$ defined in Eq. (5). $\mathcal{T}(\zeta, z)$ is zero (that means, at its absolute minimum) for the

minimizing fine field $\{z(\zeta)\}$, hence $\{z(\zeta)\}$ is also a stationary point of $\mathcal{A}_{\mathrm{FP}}$ and $\{z(\zeta)\}$ satisfies the classical FP equations of motion. Furthermore, the value of the action is the same: $\mathcal{A}_{\mathrm{FP}}(z(\zeta))=\mathcal{A}_{\mathrm{FP}}(\zeta)$ q.e.d.

Note, that the reverse statement is not always true. If the fine configuration $\{z\}$ is a solution of the equations of motion, then the coarse configuration obtained from Eq. (11) is a local minimum, but it need not be the absolute minimum that has to be found at the 
right-hand side of the FP equation (7). This mechanism prevents the existence of arbitrarily small instantons on the lattice (see below).

Using the above statement, we can now construct instanton configurations on the lattice (see also Ref. [3] for the corresponding construction in the $\mathrm{O}(3)$ nonlinear $\sigma$ model). We consider instanton configurations of the continuum on a torus. On a torus, however, there exists no exact one-instanton configuration [14]. In order to clearly separate cutoff effects from finite size effects, we have to fall back upon exact two-instanton configurations. The two-instanton configurations of $\mathrm{CP}^{1}$, embedded into $\mathrm{CP}^{N-1}$, have the form

$$
\begin{gathered}
z(w)=\mathcal{N}\left[\vec{u}+\prod_{i=1}^{2} \frac{\sigma\left(w-a_{i}\right)}{\sigma\left(w-b_{i}\right)} \vec{v}\right], \\
w=x+i y, \quad \vec{u}=(1,0,0, \ldots), \quad \vec{v}=(0,1,0, \ldots), \quad \sum_{i=1}^{k} a_{i}=\sum_{i=1}^{k} b_{i},
\end{gathered}
$$

where $\sigma(n)$ is the Weierstrass $\sigma$ function and the factor $\mathcal{N}$ ensures the correct normalization. The vectors $\vec{u}$ and $\vec{v}$ are $N$-component $\mathrm{CP}^{N-1}$ vectors which specify the orientation of the instantons in "color space". The four complex parameters $a_{1}, a_{2}, b_{1}$, and $b_{2}$ specify the size of the instantons and their position and orientation on the torus. We choose them in the form

$$
\begin{array}{ll}
a_{1}=(L / 2-\rho) i, & a_{2}=L / 2+(L / 2+\rho) i, \\
b_{1}=(L / 2+\rho) i, & b_{2}=L / 2+(L / 2-\rho) i,
\end{array}
$$

where $\rho$ is the instanton size and $L$ the size of the torus. The action $\mathcal{A}_{\text {cont }}$ of the configuration (12) is equal $2 \times 2 \pi$ irrespective of the size $\rho$ of the instantons. Next we want to construct lattice two-instanton configurations of different sizes. We first discretize the continuum two-instanton configuration on a very fine lattice, so that the cutoff effects can safely be neglected. Then we perform $k$ block transformations using Eq. (11). Under a block transformation the size of the instantons is halved. Choosing the number $k$ of block transformations and the initial size $\rho$, one can get any final size $\rho 2^{-k}$ on a coarse lattice. The 
above statement shows, that the action remains the same, unless the size of the instantons is too small, and they fall through the lattice.

We have numerically performed the above program for the $\mathrm{CP}^{3}$ model, and have measured several quantities on the finally blocked configurations. On the coarse configuration itself we measured the geometric charge, the standard action, the Symanzik improved action and the parametrization of the FP action presented in Sec. III . Performing a minimization on a multigrid with three finer levels, we measured the exact FP action and on the finest level the FP charge (cf. Sec. $\left[\mathrm{IV}\right.$ ). Because instantons in $\mathrm{CP}^{3}$ are embedded $\mathrm{CP}^{1}$ instantons, it is clear, that the results presented in Fig. 1 are practically identical to the ones obtained in the $\mathrm{O}(3)$ nonlinear $\sigma$ model [3]. The results show, that it is possible to obtain a parametrization of the FP action that performs very well for instanton configurations down to the smallest possible size on the lattice. In contrast, the standard action and also the Symanzik improved action perform quite bad, especially for small instantons. It is worth mentioning, that the range of instanton sizes, where the geometric charge differs from the FP charge, is quite narrow. For instanton configurations, the geometric charge seems to be as good as the FP charge. For general configurations created in a Monte Carlo (MC) simulation, however, there is a noticeable difference between the two charges (cf. Sec. VI).

\section{Parametrization of the FP action}

If we want to use the FP action in practical numerical simulations, we must construct a parametrization, that consists of a not too large set of local operators. Our parametrization has the form

$$
\mathcal{A}_{\mathrm{FP}}^{\mathrm{par}}(z)=-\frac{1}{2} \sum_{n, r} \rho(r) \theta_{n, n+r}^{2}+\sum_{n_{i}, n_{j}, \ldots} \text { coupling } \times \text { products of } \theta_{n_{i}, n_{j}}^{2},
$$

where $\theta_{n_{i}, n_{j}}=\arccos \left(\left|\bar{z}_{n_{i}} z_{n_{j}}\right|\right)$ is the angle between two spins. Note, that in $\mathrm{CP}^{N-1}$ models the maximal angle between two spins is $\pi / 2 ; z$ and $-z$ is the same spin, different only by a gauge transformation. There are two reasons why it is useful to use the angle $\theta_{n_{i}, n_{j}}$ 
instead of $\left(1-\left|\bar{z}_{n_{i}} z_{n_{j}}\right|^{2}\right)$ : For solutions of the equations of motion of the form $z(n)=$ $\left(\cos \theta n_{0}, \sin \theta n_{0}, 0, \ldots\right)$, the $\theta$ dependence of the action is exactly $\theta^{2}$. Moreover, if one rotates a single spin in a trivial background with all spins pointing in the same direction, the $\theta$ dependence of the action is more like $\theta^{2}$ rather than $1-\cos ^{2}(\theta)$. Figure 2 shows how well the minimized FP action is approximated even by the two first terms (nearest neighbor, diagonal) of the lowest order of the parametrization in Eq. (14). In contrast, the standard action performs very bad for large angles.

We can calculate the coefficients $\rho(r)$ of the lowest order analytically. The coefficients of the higher orders can be determined in a numerical fitting procedure.

\section{Lowest order, determination of $\rho$}

The analytic result for the lowest order term in the FP action will be valid for all $N$, as long as $N>1$. Consider on the coarse level a smooth configuration, which is weakly fluctuating around the first direction

$$
\zeta_{n_{B}}=\left(\begin{array}{c}
\sqrt{1-\left|X_{n_{B}}\right|^{2}} \\
X_{n_{B}}
\end{array}\right),
$$

where $X_{n_{B}}$ has $(N-1)$ components, and $\left|X_{n_{B}}\right| \ll 1$. With this choice we have fixed the gauge by imposing, that the first component is real and positive. Then the minimizing fine configuration will also fluctuate around the first direction and, using the same gauge fixing prescription, we make the ansatz

$$
z_{n}=\left(\begin{array}{c}
\sqrt{1-\left|V_{n}\right|^{2}} \\
V_{n}
\end{array}\right),
$$

with $\left|V_{n}\right| \ll 1$. Putting these expansions and the parametrization of the action (14) in the FP equation (77) and keeping only terms up to quadratic order in $X$ and $V$, we get the equation

$$
\begin{aligned}
& \frac{1}{2} \sum_{n_{B}, r_{B}} \rho\left(r_{B}\right) \operatorname{Re}\left(\bar{X}_{n_{B}} X_{n_{B}+r_{B}}\right)= \\
& \min _{\{V\}}\left\{\frac{1}{2} \sum_{n, r} \rho(r) \operatorname{Re}\left(\bar{V}_{n} V_{n+r}\right)+2 \kappa \sum_{n_{B}}\left|X_{n_{B}}-\frac{1}{4} \sum_{n \in n_{B}} V_{n}\right|^{2}\right\} .
\end{aligned}
$$


This equation can be solved for $\rho$ most easily by using the same technique as described in Ref. [1]. Taking into account the fact, that for the $\mathrm{CP}^{1}$ model the RG transformation goes over to the one used for the $\mathrm{O}(3)$ nonlinear $\sigma$ model, it is not astonishing, that the resulting $\rho$ is exactly the same as in Ref. [四]. Consequently, in order to have a most local action, we will also choose the free parameter $\kappa=2$ in the RG transformation Eq. (3).

\section{Higher orders for the $\mathrm{CP}^{3}$ model}

In order to have a reasonably good parametrization of the lattice action which performs well also for coarse configurations, we include higher order terms in the parametrization (14). The coefficients of these higher order terms will depend on the chosen model, and are different for each $N$. We determined them numerically for the $\mathrm{CP}^{3}$ model. To do this, we first produced about 300 configurations of lattice size 3 with a Monte Carlo program using the standard action. The configurations ranged from ones with small actions and fluctuations with small amplitudes to strongly fluctuating ones. We also included some twoinstanton configurations with radii of the order of one lattice spacing (cf. Sec. ПIIB). For every configuration we calculated the value of the FP action by minimizing the FP equation on a multigrid. Then we determined the coefficients of 30 higher order operators chosen because of their locality. Their value was determined so that the average difference between minimized and parametrized FP action was minimal.

Figure 3 illustrates the quality of the parametrization. The relative deviation from the minimized FP action is only large for very coarse configurations with large actions. For comparison, we also plot the standard action for the same configurations. Note, that here the relative deviation of the standard action from the FP action is not small, even for configurations with small amplitude fluctuations.

The resulting 32 coefficients of the parametrization (14) are given with a graphical notation of the corresponding operators in Table —. A line with two dots $\longrightarrow$ means that

the angle $\theta_{n_{1}, n_{2}}^{2}$ between the two spins at positions $n_{1}$ and $n_{2}$ of the dots enters into the 
parametrization. A graph consisting of several lines represents just the multiplication of the corresponding angles. Coefficients No. 1 and No. 4 are the only analytically calculated ones, all the other couplings were determined by the fitting procedure. The locality of the action is expressed in the fact, that it is possible to construct a good parametrization with operators consisting of spins, that lie only within a $2 \times 2$ section of the whole lattice. On the other hand, the parametrization seems to be not as local as the one found in the $\mathrm{O}(3)$ nonlinear $\sigma$ model. Some couplings of operators of higher order have quite a large value. Nevertheless, one should not give too much attention to the actual value of the couplings. A large coupling for a higher order operator does not mean, that the action is less local. Some combinations of couplings can be changed without much affecting the quality of the parametrization. In principle one would not need to keep all 32 operators in the parametrization, we just did so for reasons of completeness. Using 32 operators for an action in a MC simulation may seem to be a lot. However, compared with the expected benefit of using such an action, the additional computational effort needed seems to be reasonable.

\section{FIXED POINT TOPOLOGICAL CHARGE}

A feature of $\mathrm{CP}^{N-1}$ models common with gauge field models is the existence of topologically nontrivial solutions. In the continuum theory the topological charge $Q$ may be defined as the integral

$$
Q=\frac{i}{2 \pi} \int d^{2} x \varepsilon_{\mu \nu} \operatorname{tr}\left[P(x) \partial_{\mu} P(x) \partial_{\nu} P(x)\right]
$$

The action is related to the charge through the inequality

$$
\mathcal{A}_{\text {cont }} \geq 2 \pi|Q|
$$

For instantons the equality holds. They minimize the action, and are therefore solutions of the equations of motion.

On the lattice, however, this concept breaks down because continuity is lost. In the continuum the topological sectors are clearly separated, but on the lattice one may continu- 
ously transform a field from one topological sector to another. Furthermore, when using the standard lattice action, the scale invariance of instanton solutions is violated. The action decreases with decreasing instanton size, and configurations that violate the inequality (19) - so called "dislocations" - are possible.

In Refs. [1, 3] it was shown that the FP action admits stable instanton solutions. Furthermore, it was shown how to construct a correct charge - the FP charge - which does not admit dislocations. In this section we will proceed along the same lines as in these works, in order to construct the FP charge for the $\mathrm{CP}^{3}$ model.

\section{A. Definition of the FP charge}

We define the FP charge by means of the iterated solution of the FP equation (9). Under a RG transformation in the limit $\beta \rightarrow \infty$, an operator $\mathcal{O}(z)$ transforms into $\mathcal{O}^{\prime}(\zeta)$ on the coarse lattice as

$$
\mathcal{O}^{\prime}(\zeta)=\mathcal{O}(z(\zeta))
$$

where the spin configuration $\{z(\zeta)\}$ is the solution of the FP equation (7). The FP topological charge is obtained in the limit of infinitely many RG transformations:

$$
Q_{\mathrm{FP}}(\zeta)=\lim _{k \rightarrow \infty} Q\left(z^{(k)}(\zeta)\right)
$$

where $\left\{z^{(k)}\right\}$ is the solution of the iterated FP equation (9) on the lowest level in a $k$ level multigrid. As the configurations get smoother on each successive level, one may choose for the charge $Q$ on the lowest level any lattice discretization of the topological charge. In this paper we will use the geometric charge [15], because it is stable against small variations of the field, if the field is sufficiently smooth, and because it always gives an integer number.

In Ref. [3] it was shown, that the combination FP action and FP topological charge always obeys the inequality

$$
\mathcal{A}_{\mathrm{FP}}(\zeta) \geq 2 \pi\left|Q_{\mathrm{FP}}(\zeta)\right|
$$


Hence there are no dislocations.

In numerical simulations it is very time consuming to minimize a multigrid for every configuration. One needs a parametrization of the solution $\{z(\zeta)\}$ of the FP equation. This will be done in the next section.

\section{B. Fixed point field}

The FP field is the fine field $z^{(k)}$ in the multigrid solution of the iterated FP equation (9) as $k$ goes to infinity. If the functional dependence of the solution on the first fine level $z^{(1)}$ on $\zeta$ is known, the FP field can be evaluated by iteration. Below we give an expression for the field $z^{(1)}=z^{(1)}(\zeta)$. (In the limit $k \rightarrow \infty$ the solution $\left\{z^{(1)}\right\}$ of the iterated FP equation is identical to the solution $\{z\}$ of the FP equation.)

We make the expansions (15) and (16) for the coarse and the fine configuration. Then the solution of the FP equation leads in lowest order to the relation

$$
V_{n}=\sum_{n_{B}} \alpha\left(n, n_{B}\right) X_{n_{B}}
$$

with $\alpha$ the same as for the $\mathrm{O}(3) \sigma$ model [1, 3, 16].

To obtain this result, we have fixed the gauge in a specific way. However, we want a relation between $\{z\}$ and $\{\zeta\}$, that does not depend on the choice of the gauge fixing prescription. Furthermore, for coarse configurations we have to include higher order terms, that parametrize the dependence of $\{z\}$ on $\{\zeta\}$. For this we construct the gauge invariant composite operators $P_{n_{B}}=\zeta_{n_{B}} \otimes \bar{\zeta}_{n_{B}}$ on the coarse lattice. On the fine lattice we build matrices $Q_{n}$ by, summing over the coarse composite operators including the next to leading order terms:

$$
Q_{n}=\sum_{n_{B}} \alpha\left(n, n_{B}\right) P_{n_{B}}+\sum_{\substack{n_{\mathrm{B}} \\ m_{B}, m_{B}^{\prime}}} \beta\left(n, n_{\mathrm{B}}, m_{B}, m_{\mathrm{B}}^{\prime}\right) \theta_{m_{B}, m_{B}^{\prime}}^{2} P_{n_{B}}
$$

and define the fine field variable $z_{n}$ as the eigenvector of $Q_{n}$ with largest eigenvalue. In next order enters the angle $\theta_{m_{B}, m_{B}^{\prime}}^{2}$ between the coarse spins at sites $m_{B}$ and $m_{B}^{\prime}$, respectively. 
In order to determine the coefficients $\beta$, we used the same $\approx 300$ configurations like for the parametrization of the action, minimized the FP equation (7) and stored the resulting fine lattices. The coefficients were then determined by minimizing the difference between the minimized fine spins and the parametrization (24).

The numerical values of the coefficients $\alpha$ and $\beta$ are given in Table $\mathbb{1}$ with a graphical notation of the corresponding operators. We chose a set of 17 operators mainly because of their compactness. Numbers 1-6 are the analytically determined coefficients $\alpha$, Nos. $7-17$ are the numerically determined coefficients $\beta$. The meaning of the graphical notation of the operators is the following: The dashed lines represent a $3 \times 3$ section of the coarse lattice grid. The cross + in between indicates the position $n$ of the matrix $Q_{n}$ in Eq. (24). The little square $\mathrm{a}$ denotes the position $n_{\mathrm{B}}$ of the coarse composite operator $P_{n_{\mathrm{B}}}$, the two

connected dots $\longrightarrow$ are the positions $m_{\mathrm{B}}$ and $m_{\mathrm{B}}^{\prime}$ of the spins whose angle $\theta_{m_{\mathrm{B}}, m_{\mathrm{B}}^{\prime}}^{2}$ enters into the parametrization. Graphs obtained by trivial symmetry transformations are not drawn separately.

\section{TOPOLOGICAL SUSCEPTIBILITY}

The topological susceptibility is defined as the ratio

$$
\chi_{t}=\frac{\left\langle Q^{2}\right\rangle}{V}
$$

where $Q$ is the topological charge and $V$ is the space-time volume. In $\mathrm{CP}^{N-1}$ models it is a dimension two quantity, that vanishes to all orders in the weak coupling expansion. From the perturbative renormalization group it is expected to scale according to the two loop $\beta$ function

$$
\chi_{t} \propto\left(\frac{1}{2} \pi N \beta\right)^{4 / N} \exp (-\pi N \beta) \quad(\beta \rightarrow \infty)
$$

In order to check the continuum limit, a scaling behavior is more important to observe than the above asymptotic scaling. One additionally measures a second quantity, e.g., 
the correlation length $\xi$, and builds the dimensionless product $\chi_{t} \xi^{2}$, which should go to a constant in the continuum limit $\xi \rightarrow \infty$.

In general, cutoff effects can originate from two sources: from the discretization of the action and from the discretization of an operator. The topological charge is an operator, that strongly exhibits lattice cutoff effects, as was demonstrated in Sec. ПIIB: instantons with a radius smaller than about $0.7 a$ fall through the lattice and are lost in a Monte Carlo simulation. This fraction is large at correlation lengths of the same order as the lattice spacing, and gets smaller as the correlation length grows. One therefore expects a scaling violation, which is determined by the small instanton size distribution.

One may estimate the behavior of the expected scaling violation by using the results of a semiclassical expansion, and performing a kind of dilute instanton gas approximation. In $\mathrm{CP}^{3}$ the probability density to find a field configuration with topological charge $Q=1$ in a sphere with radius $R$ is [5]

$$
D_{1}(R)=K_{1}(4) \Lambda_{\mathrm{MS}}^{4} R^{2} \ln ^{2}\left(\Lambda_{\mathrm{MS}} R\right) \quad(R \rightarrow 0)
$$

where $\Lambda_{\mathrm{MS}}$ is the perturbative $\Lambda$ parameter and $K_{1}(4)=36.2995$ results from the integration over the instanton parameters. This result is valid for small volumes, so we can use it to estimate the probability of loosing a charge-one configuration that falls through a small lattice mesh.

We denote the charge measured on the lattice with $Q$ and the lost charge with $q$. If we assume that $Q$ and $q$ are independent, then the topological susceptibility measured on the lattice is

$$
\chi_{t}^{\text {lat }}=\frac{\left\langle Q^{2}\right\rangle}{V}=\chi_{t}^{\text {cont }}-\frac{\left\langle q^{2}\right\rangle}{V} .
$$

We make a kind of dilute gas approximation by assuming that within each lattice mesh there can be independently an instanton or an antiinstanton. The result is

$$
\frac{\left\langle q^{2}\right\rangle}{V}=2 D_{1}
$$


Identifying $R$ with the minimal size $\rho=c a$ of an instanton on the lattice $(c \simeq 0.7)$ provides

$$
\chi_{t}^{\text {lat }}=\chi_{t}^{\text {cont }}-2 K_{1}(4) \Lambda_{\mathrm{MS}}^{4} c^{2} a^{2} \ln ^{2}\left(\Lambda_{\mathrm{MS}} c a\right), \quad(a \rightarrow 0)
$$

which can be compared with the results of a Monte Carlo simulation.

\section{NUMERICAL RESULTS}

We performed Monte Carlo simulations in the $\mathrm{CP}^{3}$ model using the parametrized FP action given in Eq. (14). In order to reduce critical slowing down, we implemented a hybrid overrelaxation algorithm similar to the one described in Ref. [9]. The mass gap $m=\xi^{-1}$ was obtained from the long distance fall off of the correlation function projected to zero spatial momentum. We made a minimal $\chi^{2}$ fit to the correlation function with the function $c\left(e^{-m x}+e^{-m(L-x)}\right)$ in the interval $x \in\left[x_{\min }, L / 2\right]$ for different $x_{\min }$. The value of $m$ is taken at an expected plateau at $x_{\min } \gtrsim \xi$.

In order to avoid finite size effects, one has to make measurements in large enough volumes. We made measurements for all $\beta$ values in volumes with the ratio $L / \xi \simeq 5.5-6$ kept approximately constant. These volumes are usually large enough to totally avoid

any finite size effects. In $\mathrm{CP}^{N-1}$ models, however, the basic $z$ particles are subject to a confining potential. Thus the resulting bound states may have a radius that is larger than the correlation length. For this reason we also made measurements for some $\beta$ values in even larger volumes (with ratios $L / \xi$ up to 12 ).

The masses that are determined in the "small" volume $L / \xi \simeq 5.6$ are about $3 \%$ below the ones measured in "infinite" volume, as can be seen in Table 피. The magnitude of this finite size effect fits nicely with the one observed in Refs. [8.17]. Furthermore, we observed, that in the large volumes the determination of the mass gap showed a nice plateau behavior. Such a plateau was sometimes not very clearly seen in the small volumes. Nevertheless, we may use the results obtained in the small volume in order to look for a scaling behavior in $\chi_{t} \xi^{2}$. The actual values are spoiled by finite-size effects, but since $L / \xi$ is constant, these effects are the same for every point. 
We checked asymptotic scaling of the mass gap according to the perturbative lattice scale in two-loop approximation

$$
\Lambda_{L}^{(2)}=\left(\frac{1}{2} \pi N \beta\right)^{2 / N} \exp \left(-\frac{1}{2} \pi N \beta\right)
$$

In Fig. 1 we show the ratio $m / \Lambda_{L}^{(2)}$ versus the correlation length, with masses determined in "small" volumes $L / \xi \simeq 5.6$ and also some measurements in "large" volumes. The most striking observation is that the ratio approaches a constant value $m / \Lambda_{L}^{(2)}=8.1(1)$ (this value is obtained in "large" volumes), and that its final value is attained already at a quite short correlation length. Such a precocious asymptotic scaling has not been stated for other lattice actions. With the standard action, for example, one does not even see an asymptotic scaling at correlation lengths $\xi \sim 50$ [9, 10]. Note that the asymptotic scaling behavior for the FP action is a purely phenomenological observation that has not been expected theoretically. The FP action was constructed nonperturbatively in order to reduce cutoff effects. That the mass gap scales with the perturbative $\Lambda$ parameter is just an additional, unexpected benefit. We furthermore observe, that the value of the ratio $m / \Lambda_{L}^{(2)}$ is remarkably small. From this we conclude, that $\Lambda_{\mathrm{FP}}$ is much closer to the continuum scale than $\Lambda_{\mathrm{ST}}$.

We determined the topological charge, using both the geometric definition and the definition of the FP charge given in Sec. IV. For the measurement of the FP charge we used the geometric charge on a finer lattice of the multigrid with the Monte Carlo generated lattice as coarsest level. In order to determine the configuration $z(\zeta)$ on the first finer level, one can either minimize the FP equation (which is very time consuming) or use the parametrization of the dependence on $\zeta$, given in Sec. IVB. We denote the corresponding charges $Q_{\text {coarse }}$

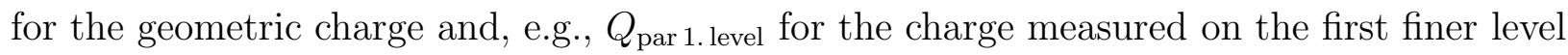
using the parametrization of the fine field.

For some $\beta$ values we compared the results of using the parametrization on a finer level and of minimizing on a multigrid. The results were found to be consistent within the statistical errors, as is reported in Table III. This shows that the parametrization performs well for typical configurations occurring in a Monte Carlo simulation. We wanted to be sure, 
that it is sufficient to measure the charge only on the first finer level. As a test we calculated for one $\beta$ value also the charge on the second finer level. The result presented in Table III shows, that the values on the first and the second finer level were found to be consistent within the statistical errors. This means that the process of going to a lower level is already stable at that stage, and that it is sufficient to calculate the fine field only on the first finer level. This is not unexpected, since we use a geometric definition of the topological charge at a lower level. Therefore, we always get an integer number for the charge, and the charge has to change abruptly when going to a finer level. The field on the first finer level is then (in most cases) smooth enough to yield already the FP topological charge.

Table III shows the effect of using different definitions of the lattice topological charge. On the coarse level the value of $\left\langle Q_{\text {coarse }}^{2}\right\rangle$ is higher than the one obtained with the FP charge (on the first finer level). This can be explained with dislocations which contribute to the geometric charge. They have the effect that one overestimates the amount of topological excitation.

In Fig. 5 we show the results of the scaling test for the dimensionless quantity $\chi_{t} \xi^{2}$, measured in volumes with a ratio $L / \xi \simeq 5.6$. One clearly sees the expected raise at small correlation lengths, which is due to lost small instantons in this region. At correlation length $\xi \simeq 10$ this effect is already saturated. The measurements in "small" volumes using the FP charge (i.e., $Q_{\text {par 1. level }}$ ) show the expected scaling plateau at a value of about $\chi_{t} \xi^{2} \simeq 0.071$ (c.f. Fig. 5). The measurements using the geometric definition of the topological charge give somewhat larger values but they seem to converge to the value obtained from the FP topological charge for larger correlation lengths - these measurements do not show the same nice scaling behavior as the measurements using the FP charge. This is also what is to be expected in the $\mathrm{CP}^{3}$ model if dislocations contribute to the geometric charge. In the continuum limit the effect of these dislocations is suppressed, but they still contribute at the considered correlation lengths.

We can now use the four measurements in "large" volumes, with $Q_{\text {par 1. level for the topo- }}$ logical charge and perform a fit to the data with Eq. (30), in order to extrapolate to the 
continuum value. We obtain the value $\chi_{t} \xi^{2}=0.070(2)$.

Let us compare our numerical value with previous determinations and with results from the large $N$ expansion [4]. To leading order in $1 / N$ one gets

$$
\chi_{t} \xi^{2}=\frac{3}{4 \pi N}+O\left(1 / N^{5 / 3}\right) \simeq 0.06 \quad(\text { for } N=4) .
$$

This value is comparable with the value we got at the scaling plateau. However, the correction to the leading order is large in Eq. (32) and the agreement with numerical results occurs at chance at $N=4$. The numerical results of Ref. [8] (who use actions with an explicit gauge field) - quoting $\chi_{t} \xi^{2} \simeq 0.06$ with an uncertainty of $10-20 \%$ - are in quite good correspondence with our result. Measurements using the standard action and the geometric definition of the topological charge (Refs. [9:10]) lead to a value roughly twice as large as ours, but with large scaling violations at large correlation lengths - this is probably the effect of dislocations which still contribute at the considered correlation lengths.

\section{CONCLUSIONS}

In $\mathrm{CP}^{N-1}$ models it is possible to define a FP action and a FP topological charge. The FP action is the fixed point of an exact $\mathrm{RG}$ transformation. It is a classically perfect action and possesses scale invariant instanton solutions. The definition of the FP topological charge is based on the FP field operator. Both, the FP action and the FP field can be evaluated to any precision desired on a sufficiently large multigrid. It can be shown that the FP topological charge together with the FP action have no lattice defects.

It is profitable to use the FP action and the FP topological charge in numerical simu-

lations. For this purpose, we have parametrized for the $\mathrm{CP}^{3}$ model the $\mathrm{FP}$ action and the field solution of the FP equation which is iterated to obtain the FP field. We used these parametrizations in $\mathrm{MC}$ simulations of the $\mathrm{CP}^{3}$ model. We find two main results. First, the mass gap unexpectedly scales according to the perturbative lattice scale. Second, the dimensionless quantity $\chi_{t} \xi^{2}$ raises as expected at small correlation lengths, and reaches a scaling plateau at $\chi_{t} \xi^{2}=0.070(2)$. 
For the future, it would be interesting and profitable to use the parametrized FP action

for further $\mathrm{MC}$ simulations, for example in order to investigate the spectrum of $\mathrm{CP}^{N-1}$ models.

\section{ACKNOWLEDGMENTS}

I would like to thank P. Hasenfratz, F. Niedermayer, M. Blatter, U.-J. Wiese, A. Papa, and P. Kunszt for helpful discussions. This work was supported in part by the Schweizerischer Nationalfonds. 


\section{REFERENCES}

[1] P. Hasenfratz and F. Niedermayer, Nucl. Phys. B414, 785 (1994), hep-lat/9308004;

P. Hasenfratz, Nucl. Phys. B (Proc. Suppl.) 34B, 3 (1994); F. Niedermayer, ibid., 513.

[2] T. DeGrand, A. Hasenfratz, P. Hasenfratz, F. Niedermayer, Nucl. Phys. B454, 587 (1995), hep-lat/9506030; B454, 615 (1995), hep-lat/9506031; F. Farchioni, P. Hasenfratz, F. Niedermayer, A. Papa, ibid.,638, hep-lat/9506032.

[3] M. Blatter, R. Burkhalter, P. Hasenfratz and F. Niedermayer, Nucl. Phys. B (Proc. Suppl.) 42B 799 (1995), hep-lat/9411068; Phys. Rev. D 53, 923 (1996), heplat/9508028.

[4] M. Lüscher, Nucl. Phys. B200[FS4], 61 (1982).

[5] P. Schwab, Phys. Lett. 118B, 373 (1982); 126B, 241 (1983); M. Lüscher, Nucl. Phys. B205[FS5], 483 (1982).

[6] A. Jevicki, Nucl. Phys. B127, 125 (1977); D. Förster, ibid. B130, 38 (1977); V.A. Fateev, I.V. Frolov and A.S. Schwarz, ibid. B154, 1 (1979); B. Berg and M. Lüscher, Comm. Math. Phys. 69, 57 (1979).

[7] K. Jansen and U.-J. Wiese, Nucl. Phys. B370 762 (1992).

[8] M. Campostrini, P. Rossi and E. Vicari, Phys. Rev. D 46, 2647 (1992); D 46, 4643 (1992), hep-lat/9207032.

[9] U. Wolff, Phys. Lett. B 284, 94 (1992), hep-lat/9205001.

[10] M. Hasenbusch and S. Meyer, Phys. Rev. D 45, 4376 (1992); Phys. Lett. B 299, 293 (1993).

[11] A.C. Irving and C. Michael, Nucl. Phys. B371, 521 (1992).

[12] A. D’Adda, M. Lüscher and P. Di Vecchia, Nucl. Phys. B146, 63 (1978). 
[13] P. Di Vecchia, A. Holtkamp, R. Musto, F. Nicodemi and R. Pettorino, Nucl. Phys. B190[FS3], 719 (1981).

[14] J.-L. Richard and A. Rouet, Nucl. Phys. B211, 447 (1983).

[15] B. Berg and M. Lüscher, Nucl. Phys. B190[FS3], 412 (1981).

[16] T. L. Bell and K. Wilson, Phys. Rev. B 10, 3935 (1974).

[17] P. Rossi and E. Vicari, Phys. Rev. D 48, 3869 (1992). 


\section{FIGURES}

FIG. 1. Actions and charge of instantons with radii of the order of one lattice spacing.

FIG. 2. Actions of configurations with one spin rotated against a trivial background.

FIG. 3. Scatter plot of the actions of the configurations used for the fit.

FIG. 4. Asymptotic scaling test for the mass in the $\mathrm{CP}^{3}$ model.

FIG. 5. Scaling test for the topological susceptibility. These are the results measured in "small" volumes $L / \xi \simeq 5.6$. The larger error bars at some results from the first finer level are due to the fact that we performed less measurements where we also measured the FP charge. 


\section{TABLES}

\begin{tabular}{|c|c|c|c|c|c|c|c|c|}
\hline No. & Type & Coupling & No. & Type & Coupling & No. & Type & Coupling \\
\hline 1 & $\bullet$ & -0.61884 & 2 & $\Longleftrightarrow$ & -0.05381 & 3 & & 0.20023 \\
\hline 4 & & -0.19058 & 5 & & -0.01892 & 6 & & -0.06735 \\
\hline 7 & & 0.01455 & 8 & & -0.25328 & 9 & & 0.07099 \\
\hline 10 & & 0.04334 & 11 & & 0.02704 & 12 & & -0.12660 \\
\hline 13 & & 0.06787 & 14 & & 0.18327 & 15 & & 0.13297 \\
\hline 16 & & -0.28036 & 17 & & -0.00174 & 18 & & 0.26017 \\
\hline 19 & & 0.11006 & 20 & & 0.01396 & 21 & & 0.09222 \\
\hline 22 & & -0.02530 & 23 & & 0.52163 & 24 & & -0.05146 \\
\hline 25 & & -0.06314 & 26 & & -0.29456 & 27 & & -0.04937 \\
\hline 28 & & 0.17930 & 29 & & -0.15733 & 30 & & -0.15941 \\
\hline
\end{tabular}




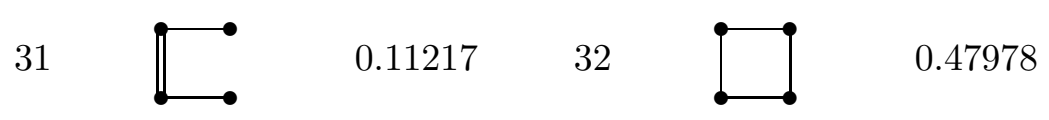

TABLE I. Couplings used for the FP action.

\begin{tabular}{|c|c|c|c|c|c|c|c|c|}
\hline No. & Type & Coeff. & No. & Type & Coeff. & No. & Type & Coeff. \\
\hline 1 & & 0.59497 & 2 & & 0.15621 & 3 & & 0.08300 \\
\hline 4 & & 0.00942 & 5 & 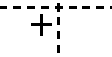 & -0.00171 & 6 & & -0.00668 \\
\hline
\end{tabular}

7

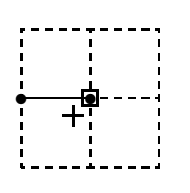

$-0.03600 \quad 8$

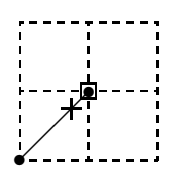

$-0.03104 \quad 9$

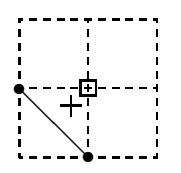

$-0.11141$

10

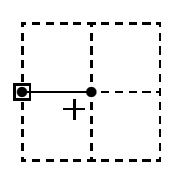

0.02788

11

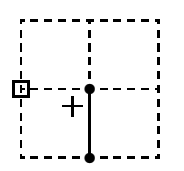

$-0.01814 \quad 12$

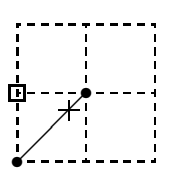

$-0.01660$

13

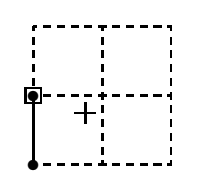

0.01600

14

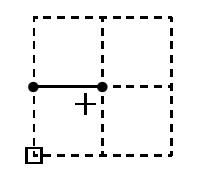

$-0.01311$

15

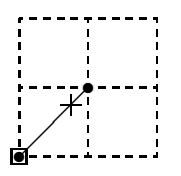

0.03643

16

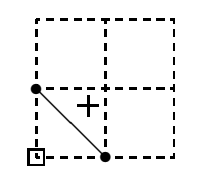

$-0.02649$

17

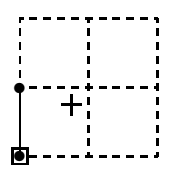

0.01849 


\begin{tabular}{|c|c|c|c|c|c|c|c|c|}
\hline$N \beta$ & $\mathrm{L}$ & $\xi$ & $\left\langle Q_{\text {coarse }}^{2}\right\rangle$ & $\left\langle Q_{\min 1 . ~ l e v e l}^{2}\right\rangle$ & $\left\langle Q_{\text {par 1. level }}^{2}\right\rangle$ & $\left\langle Q_{\text {par 2. level }}^{2}\right\rangle$ & $\chi_{\min 1 . \text { level }}^{t} \xi^{2}$ & $\chi_{\text {par 1. level }}^{t} \xi^{2}$ \\
\hline 1.4 & 6 & $0.9972(27)$ & $1.0981(66)$ & $1.008(17)$ & & & $0.0278(5)$ & \\
\hline 2.1 & 12 & $2.0085(68)$ & $1.8950(98)$ & $1.758(22)$ & & & $0.0492(7)$ & \\
\hline 2.45 & 18 & $3.044(14)$ & $2.270(13)$ & $2.157(28)$ & $2.121(26)$ & $2.114(44)$ & $0.0617(10)$ & $0.0607(9)$ \\
\hline 2.45 & 34 & $2.9500(71)$ & $8.235(46)$ & & $7.794(44)$ & & & $0.0587(4)$ \\
\hline 2.7 & 24 & $4.267(19)$ & $2.272(16)$ & $2.152(31)$ & & & $0.0680(12)$ & \\
\hline 3.0 & 36 & $6.422(27)$ & $2.373(16)$ & & $2.282(34)$ & & & $0.0726(12)$ \\
\hline 3.0 & 54 & $6.241(23)$ & $5.477(39)$ & & $5.208(37)$ & & & $0.0696(7)$ \\
\hline 3.0 & 76 & $6.244(21)$ & $11.01(11)$ & & $10.51(11)$ & & & $0.0709(9)$ \\
\hline 3.2 & 48 & $8.569(26)$ & $2.414(17)$ & $2.360(42)$ & $2.277(24)$ & & $0.0752(14)$ & $0.0726(9)$ \\
\hline 3.5 & 74 & $13.063(71)$ & $2.410(31)$ & & $2.304(29)$ & & & $0.0718(12)$ \\
\hline 3.5 & 94 & $12.897(73)$ & $3.945(49)$ & & $3.786(48)$ & & & $0.0713(12)$ \\
\hline 3.79 & 110 & $19.945(83)$ & $2.217(23)$ & & $2.138(36)$ & & & $0.0703(13)$ \\
\hline 3.79 & 200 & $19.43(13)$ & $7.61(13)$ & & $7.29(12)$ & & & $0.0688(15)$ \\
\hline 3.95 & 150 & $24.92(15)$ & $2.607(35)$ & & $2.528(34)$ & & & $0.0698(13)$ \\
\hline
\end{tabular}

TABLE III. Results of MC simulations. 


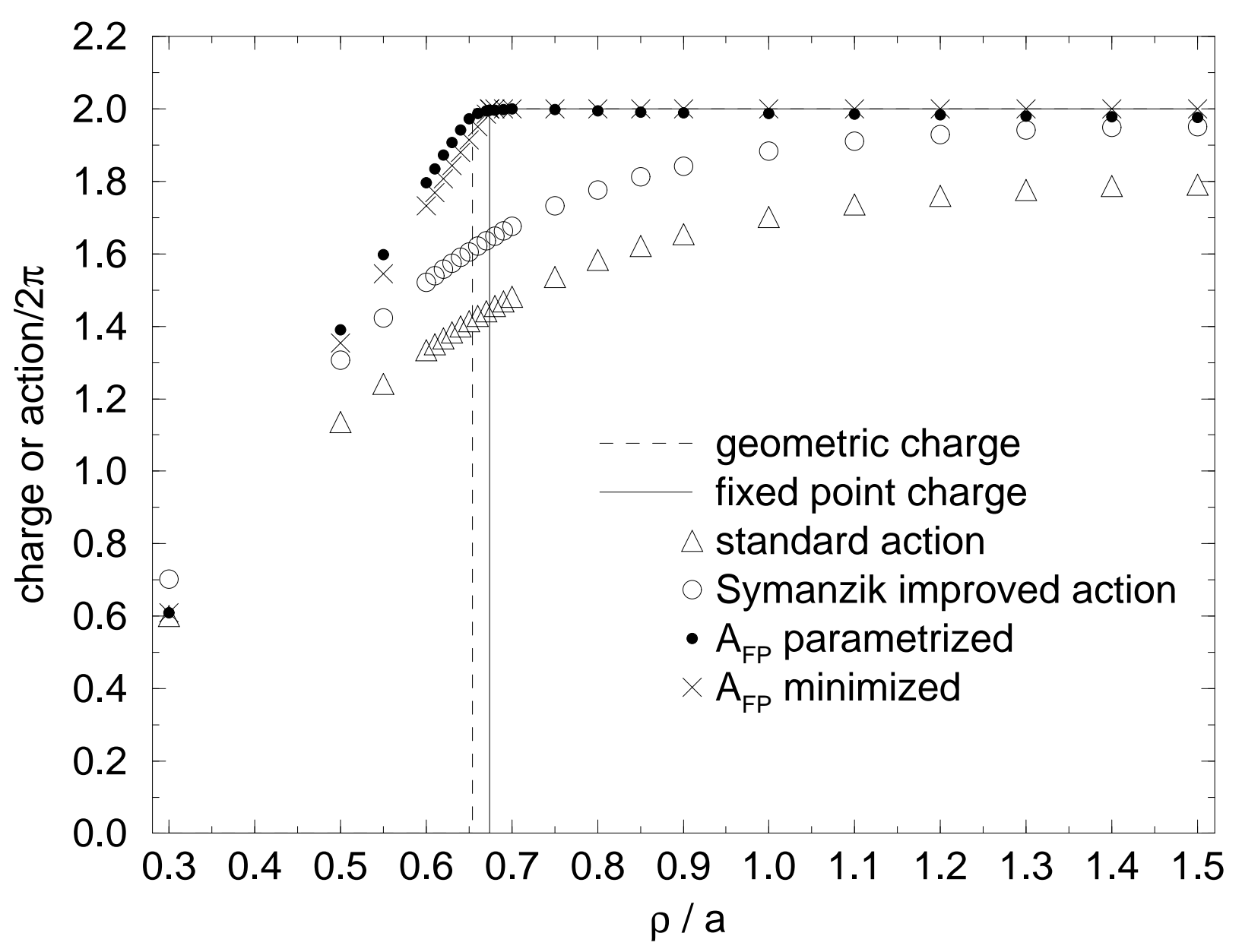




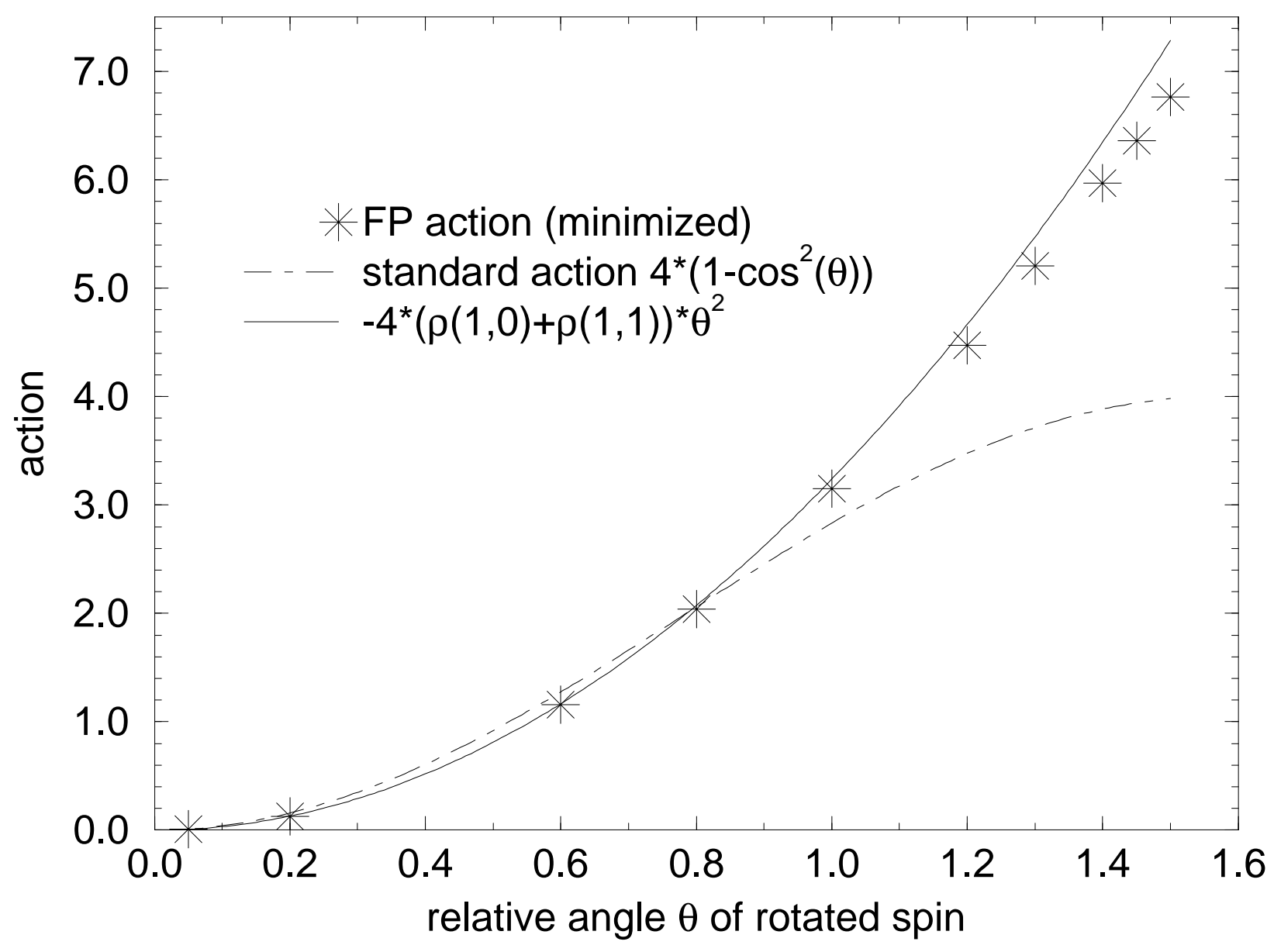




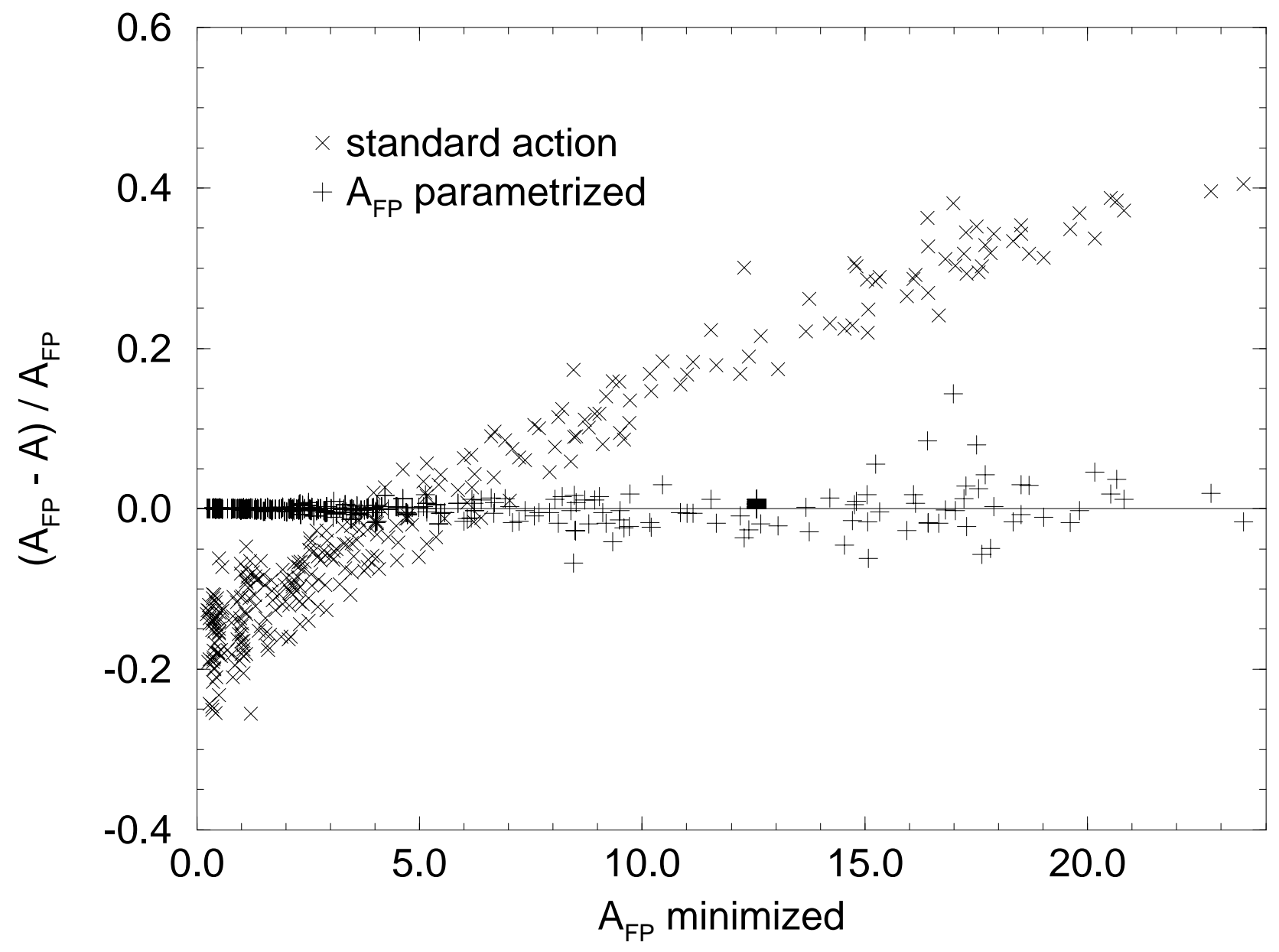




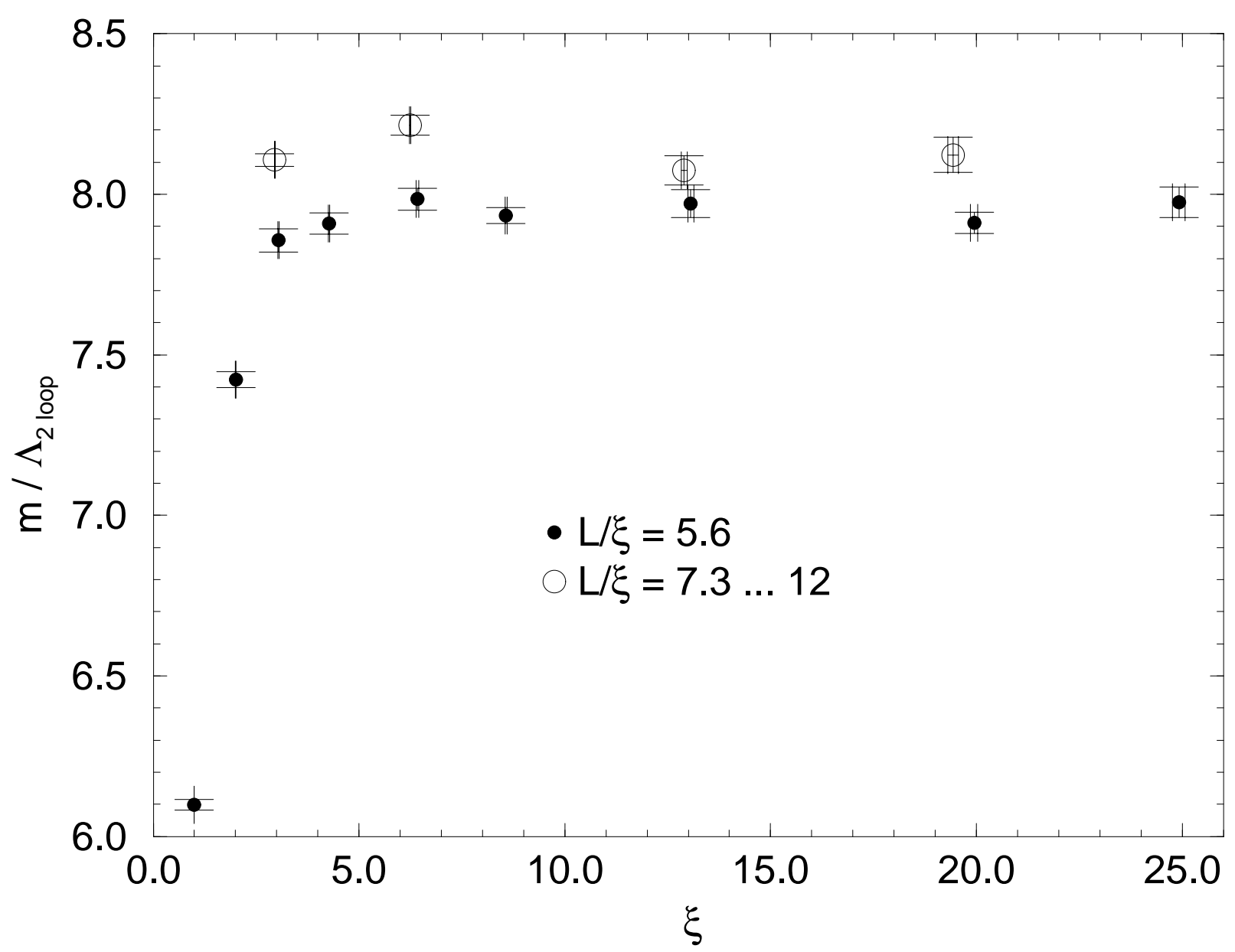




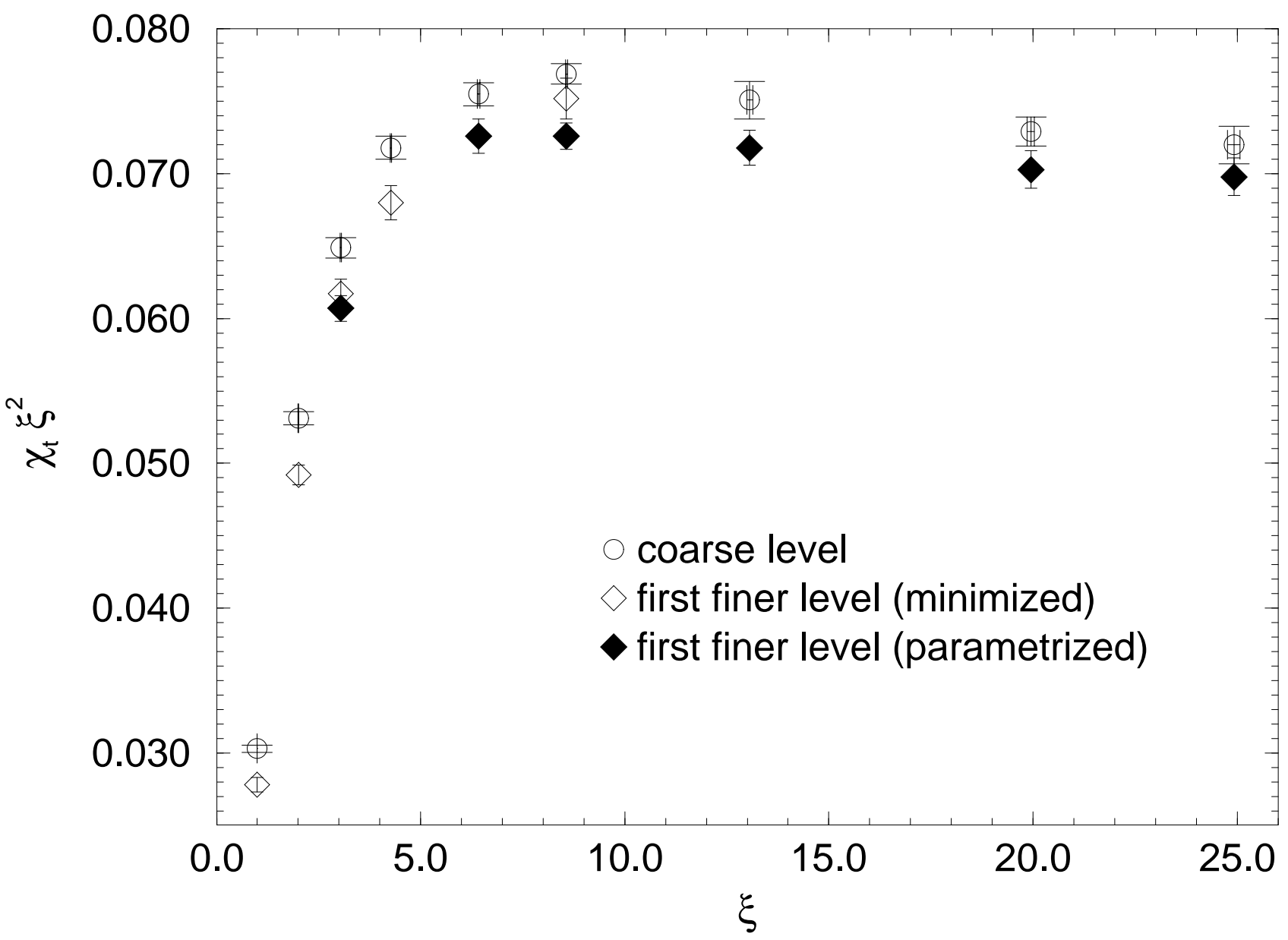

